\title{
(2) OPEN ACCESS \\ Risk factors associated with higher pain levels among pediatric burn patients: a retrospective cohort study
}

\author{
Maleea D Holbert (D) ,'2 Roy M Kimble, ${ }^{1,3}$ Lee V Jones, ${ }^{4,5}$ Samiul H Ahmed, ${ }^{2}$ \\ Bronwyn R Griffin ${ }^{1,6}$
}

${ }^{1}$ Centre for Children's Burns and Trauma Research, Queensland Health Centre for Children's Health Research, South Brisbane, Queensland, Australia 2Faculty of Medicine, The University of Queensland, Brisbane, Queensland, Australia ${ }^{3}$ Pegg Leditschke Paediatric Burns Centre, Queensland Children's Hospital, South Brisbane, Queensland, Australia ${ }^{4}$ Research Methods Group, Queensland University of Technology, Kelvin Grove, Queensland, Australia ${ }^{5}$ Faculty of Health, Centre for Healthcare Transformation, Queensland University of Technology, Kelvin Grove, Queensland, Australia

${ }^{6}$ Faculty of Health, School of Nursing, Queensland University of Technology, Kelvin Grove, Queensland, Australia

\section{Correspondence to} Maleea D Holbert, Centre for Children's Burns and Trauma Research, Queensland Health Centre for Children's Health Research, South Brisbane, QLD 4101, Australia;

m.holbert@uq.edu.au

Received 17 May 2020 Revised 9 October 2020 Accepted 14 October 2020 Published Online First 9 November 2020

\section{ABSTRACT}

Introduction There is an absence of evidence regarding predictors of moderate to severe pain in children undergoing acute burn treatment. This investigation aimed to determine if relationships existed between patient and clinical characteristics, and pain at first dressing change for children with acute burn injuries. Methods A retrospective cohort investigation was conducted using clinical data from pediatric burn patients treated at the Queensland Children's Hospital, Brisbane, Australia. Data extracted included patient and burn characteristics, first aid, and follow-up care. Observational pain scores were categorized into three groups (mild, moderate, and severe pain), and bivariate and multivariable relationships were examined using proportional odds ordinal logistic regression. Data from 2013 pediatric burns patients were extracted from the database.

Results Factors associated with increased odds of procedural pain included: hand burns (OR 1.7, 95\% Cl 1.3 to $2.1, p<0.001)$, foot burns (OR $1.5,95 \% \mathrm{Cl} 1.1$ to $2.1, p<0.01$ ), baseline pain (OR $5.5,95 \% \mathrm{Cl} 2.8$ to $10.8, p<0.001)$, deep dermal partial-thickness injuries (OR 7.9, 95\% Cl 4.0 to $15.6, p<0.001$ ), increased burn size (OR $1.1,95 \% \mathrm{Cl} 1.0$ to $1.2, \mathrm{p}<0.01)$, four or more anatomical regions burned (OR 3.6, $95 \% \mathrm{Cl} 1.5$ to 8.6 , $\mathrm{p}<0.01$ ), initial treatment at a non-burns center (OR $1.8,95 \% \mathrm{Cl} 1.4$ to $2.3, \mathrm{p}<0.001)$, and time to hospital presentation (OR $0.9,95 \% \mathrm{Cl} 0.8$ to $0.9, \mathrm{p}<0.001$ ) These burn characteristics are associated with increased odds of moderate to severe procedural pain during a child's first dressings change.

Discussion It is recommended that patients presenting with one or more of the aforementioned factors are identified before their first dressing change, so additional pain control methods can be implemented.

\section{INTRODUCTION}

Literature indicates that children are one of the most vulnerable populations for burn injuries-in particular children under the age of $5 .^{12}$ Pain is a major issue following a burn, and research suggests that pain from burn injuries continues to be undertreated. ${ }^{3}$ A better understanding of factors that influence pain in pediatric burn patients is vital to establish effective pain management guidelines for children with burn injuries-and to recognize patients who will require a more aggressive and targeted approach to pain management during their burn treatment. Comprehensive management of burn wounds includes a challenging spectrum of acute, background, breakthrough, and procedural pain. ${ }^{45}$ Optimizing pain management for pediatric burn patients is critical, as untreated pain can lead to prolonged wound re-epithelization. ${ }^{67}$ In addition, inadequate pain management can lead to the development of maladaptive long-term outcomes such as chronic pain conditions ${ }^{8}$ and post-traumatic stress symptoms. ${ }^{89}$ While previous studies have investigated factors that influence burn wound re-epithelialization in children, few have examined factors that increase procedural pain scores in a pediatric burn population. This research aimed to examine the association between patient and burn characteristics and pain experienced during a child's first dressing change-aiming to determine risk factors associated with moderate to severe procedural pain in children with acute burn injuries. Four variables were hypothesized to be associated with increased procedural pain scores during a child's first burn dressing change-burn size, the number of burns sustained to different anatomical regions, unilateral/bilateral foot burns, and burn mechanism.

\section{METHODS}

\section{Design and setting}

A retrospective cohort investigation was conducted using clinical data from the Queensland Pediatric Burns Registry, which captures all patients who attend the Pegg Leditschke Children's Burns Outpatient Department (OPD) at the Queensland Children's Hospital (QCH) for burn wound treatment. This registry records all pediatric outpatients with a burn treated at the QCH, from first aid and acute care through to discharge from the burns service or referral to scar management. The QCH, located in South Brisbane, Australia, serves as the major catchment area for pediatric burns occurring in Queensland and Northern New South Wales, treating over 1200 pediatric patients with burn injuries per annum. Data from February 2016 to July 2019 were extracted and included for analysis $(\mathrm{n}=2013)$.

\section{Patients and procedures}

Informed consent was obtained from each parent/ caregiver, as well as child assent if age appropriate. Structured interviews were conducted with all parents/caregivers and patients who consented to have their clinical information recorded during their first presentation to the Burns OPD at the QCH. Patient demographic details, burn mechanism, first aid interventions, and time to presentation were obtained during these structured interviews. As 


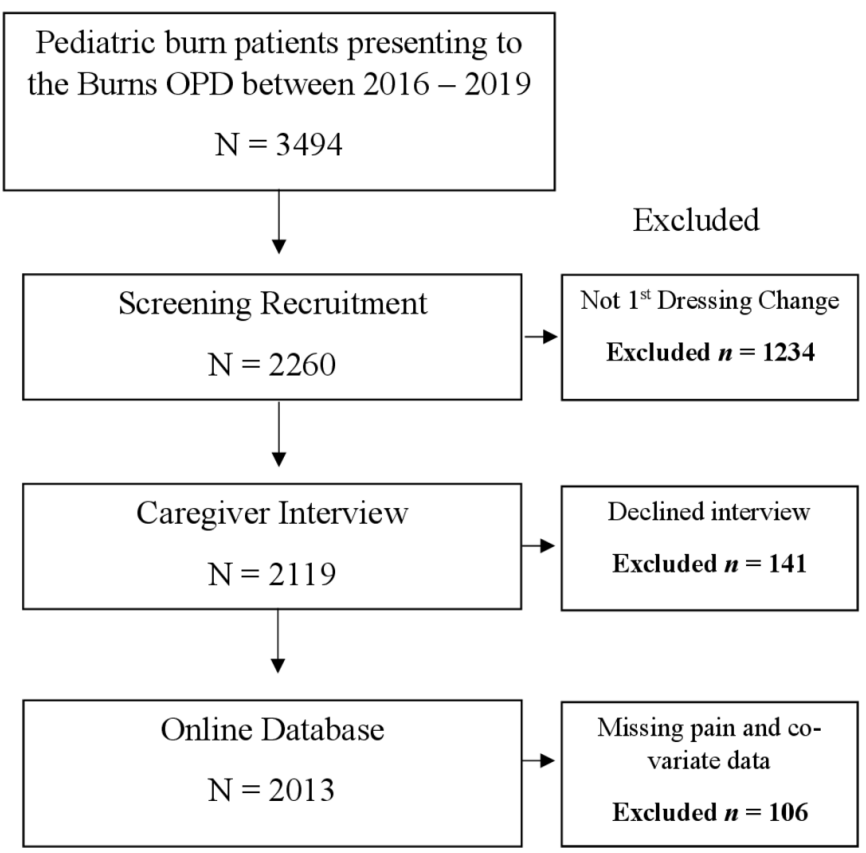

Figure 1 Participant flow diagram. N, number of participants; OPD, outpatient department.

part of routine clinical practice, all patients treated at the Burns OPD for a dressing change have their pain scores recorded in the waiting room prior to pre-medication and silver dressing removal (1. Pre-dressing removal), during debridement and wound cleaning (2. Peak procedural pain), and following the application of new silver dressings if the child's burns have not re-epithelialized (3. Post-dressing application). Specialized burns nursing staff assessed pain at these time points using the Face, Legs, Activity, Cry, and Consolability (FLACC) rating scale. This scale consists of five behavioral categories, each of which are scored on a $0-2$ point scale, giving a total score which ranges from 0 to 10 . The FLACC scale has been shown to have good intra-rater and inter-rater agreement and is considered a feasible and valid pain assessment tool in children. ${ }^{10-12}$ Peak procedural pain, assessed using the FLACC, was the dependent variable of interest for this investigation.

Demographic information collected from structured patient and parent/caregiver interviews, and clinical data from treating medical and nursing staff, were entered into the Queensland Pediatric Burns Registry for each pediatric burn patient seen in the Burns OPD. Data from 3494 patients were extracted from the Queensland Pediatric Burn Registry from 2016 to 2019. Data extraction for this investigation occurred in August 2019. Where possible, missing data within the database were completed using patient's electronic medical records. Patients were excluded from the investigation if parents/caregivers declined to have their child's information entered into the database, or if procedural pain scores or co-variable data were not documented during the patient's first dressing change in the Burns OPD (see figure 1).

\section{Variable measures}

In medical and clinical research settings, pain is often measured using an 11-point rating scale (ranging from 0 to 10). Treatment guidelines, however, such as the WHO Cancer Pain Guidelines, ${ }^{13}$ are often developed based on categorical ratings of pain (ie, mild, moderate, and severe). In this investigation, pain was defined in accordance with the optimal classification scheme for average pain as ${ }^{14}$
1. Mild Pain $=0-2$.

2. Moderate $=3-6$.

3. Severe $=7-10$.

Nursing observational pain scores (for peak procedural pain) assessed using the FLACC during the child's first burns dressing change were grouped into three categories (mild, moderate, and severe) indicating ordered levels of pain. Peak procedural pain categorized into mild, moderate, and severe groups served as the primary outcome for this investigation. Patients who reported mild, moderate, and severe pain during their first dressing change in the Burns OPD were then examined for influencing factors related to clinical features. The following variables were extracted from the database: age, sex, residential postcode, place of birth, Aboriginal and Torres Strait Islander (ATSI) status, burn depth, burn total body surface area (TBSA), anatomical location of the burn, number of anatomical regions affected, burn mechanism, burn sub-mechanism, first aid, pre-hospital care, time taken to present to hospital, peak procedural pain (measured using the FLACC rating scale) at first dressing change, and number of analgesic medications administered. Socioeconomic status was determined using the patient's residential post code and associated Socio-Economic Index of Areas (SEIFA) generated using Census of Population and Housing information from the Australian Bureau of Statistics. Patients who experienced moderate to severe pain during their first change of dressing for their burn injuries were identified within the database.

\section{Statistical analysis}

Proportional odds ordinal logistic regression was used to explore associations between patient and burn characteristics and peak procedural pain (categorized into mild, moderate, and severe groups) during a child's first change of dressing. Bivariate models were run to generate crude odds, which were then used to construct a multivariable model. All predictors with a $\mathrm{p}$ value of less than 0.2 (from the bivariate model) were entered into the initial multivariable model. Following this, variables not attaining statistical significance $(p<0.05)$ were removed from the initial multivariable model one at a time. Variables in the final model were included if significant $(p<0.05)$. Descriptive statistics were calculated for each of the identified variables. Data were described as frequencies, percentages, and medians (quartiles) for non-parametric data. Analysis was performed using SPSS software V.23. Values of $p<0.05$ were considered statistically significant.

\section{RESULTS}

\section{Participant characteristics}

Clinical and demographic details for the 2013 pediatric burn patients are reported in table 1 . The median participant age was 3 (1-7) years. Median TBSA was equal to $1 \%$ with a minimum and maximum range between $0.5 \%$ and $23 \%$ within the cohort sample (see online appendix 1 for detailed TBSA data and online appendix 2 for burn mechanism breakdown). Bivariate results (crude odds) are presented in table 2.

\section{Main results}

Multivariable results are presented in figure 2. Because this was an exploratory analysis, we did not adjust for multiple comparisons. Results show the odds of having (one category) increased pain was higher in children with full-thickness burns (OR 5.7, 95\% CI 2.1 to $15.3, \mathrm{p}<0.01$ ), DDPT burns (OR 7.9, 95\% CI 4.0 to $15.6, \mathrm{p}<0.001$ ), and SPT injuries (OR 3.3, 95\% CI 1.7 to 6.2 , $\mathrm{p}<0.001)$ in comparison with patients with superficial burns. 


\section{Original research}

Table 1 Characteristics of study participants according to pain category

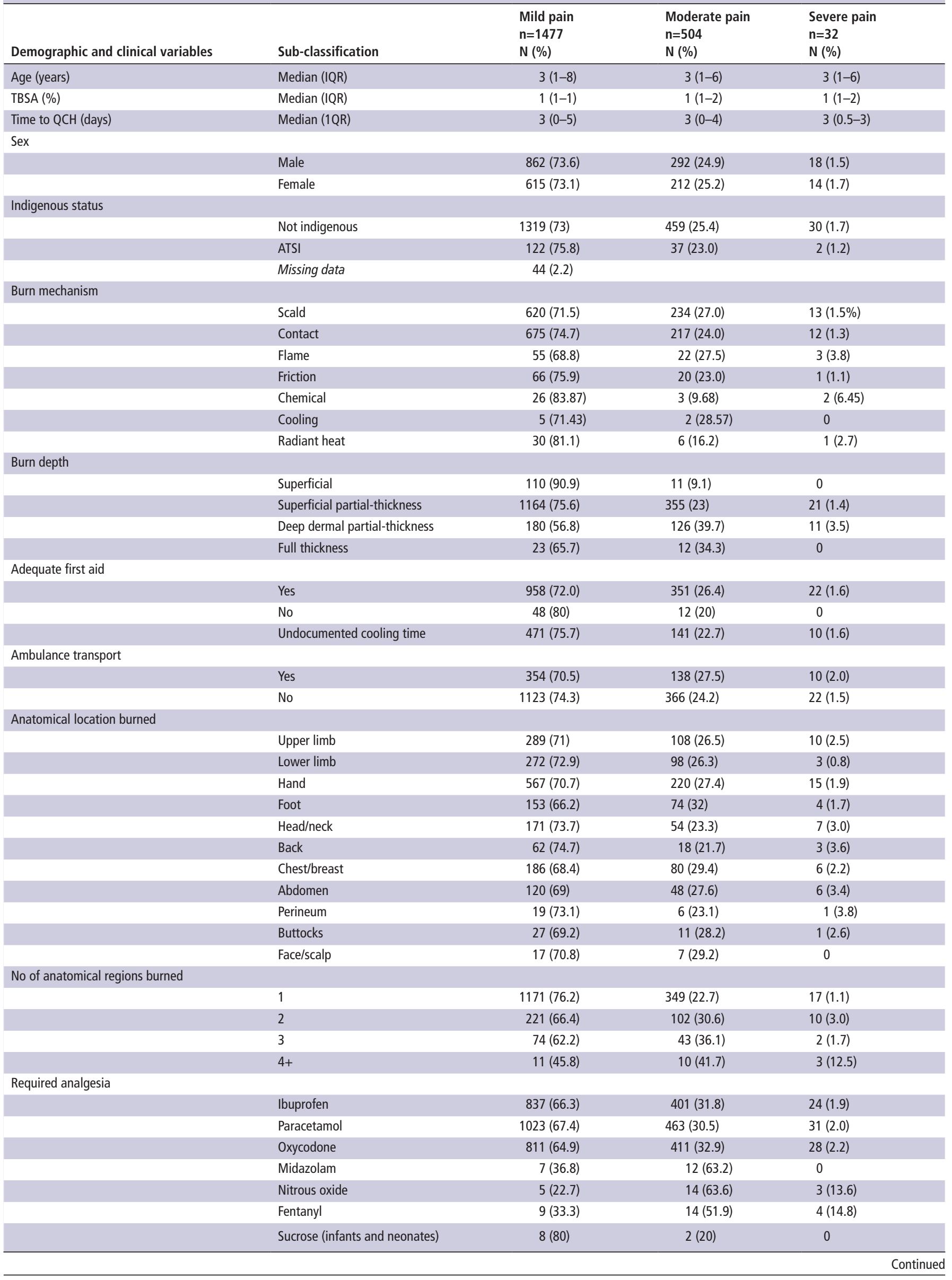




\begin{tabular}{|c|c|c|c|c|}
\hline Demographic and clinical variables & Sub-classification & $\begin{array}{l}\text { Mild pain } \\
n=1477 \\
N(\%)\end{array}$ & $\begin{array}{l}\text { Moderate pain } \\
n=504 \\
N(\%)\end{array}$ & $\begin{array}{l}\text { Severe pain } \\
n=32 \\
N(\%)\end{array}$ \\
\hline \multicolumn{5}{|l|}{ Burns OPD referral source } \\
\hline & Other hospital & $785(70.1)$ & $316(28.2)$ & $19(1.7)$ \\
\hline & Self-referral & $24(80)$ & $4(13.3)$ & $2(6.7)$ \\
\hline Baseline pain (0-10 scale) & Median (IQR) & $0(0-0)$ & $0(0-0)$ & $0(0-0)$ \\
\hline Peak pain (0-10 scale) & Median (IQR) & $1(0-1)$ & $3(3-4)$ & $8(7-9.8)$ \\
\hline
\end{tabular}

$\mathrm{N}$, number of participants; TBSA, total body surface area; OPD, outpatient department; $\mathrm{QCH}$, Queensland Children's Hospital; ED, emergency department.

Children with DDPT burns had almost eight times greater odds of increased pain at their first dressing change. In addition to burn depth, children with burns affecting their hands (OR 1.7, $95 \%$ CI 1.3 to $2.1, \mathrm{p}<0.001$ ) had greater odds of increased pain compared with pediatric patients with burns to other anatomical regions (upper limb, lower limb, chest, abdomen, and back). Furthermore, patients with foot burns (OR 1.5, 95\% CI 1.1 to $2.1, \mathrm{p}<0.01)$ had greater odds of increased pain at their first dressing change in comparison with children without foot burns.

Pediatric burn patients had higher odds of increased pain with each $1 \%$ increase in TBSA (OR $1.1,95 \%$ CI 1.0 to $1.2, \mathrm{p}<0.01$ ). Likewise, pediatric patients with burns to four or more anatomical regions (OR 3.6, 95\% CI 1.5 to $8.6, \mathrm{p}<0.01$ ) had almost four times greater odds of increase pain (ie, moving from mild to moderate pain, or moderate to severe pain) in comparison with patients with one anatomical location burned. Children who reported baseline pain (pain pre-dressing removal) had six times greater odds of moving up a pain group (ie, moving from the mild to moderate pain group, or moderate to severe pain) in comparison with children who did not report baseline pain (OR 5.5, 95\% CI 2.8 to $10.8, \mathrm{p}<0.001)$.

Furthermore, patients who first presented to a non-specialist hospital or medical center (OR 1.8, 95\% CI 1.4 to 2.3 , p < 0.001) for acute burn treatment (and were later referred to the $\mathrm{QCH}$ Burns OPD) had almost two times greater odds of higher procedural pain during their first change of dressing compared with patients who presented to the QCH ED (a burns specialist, trauma-verified hospital), or were admitted as an inpatient at the QCH prior to their Burns OPD referral. Pediatric patients who presented to the QCH soon after sustaining their burn had lower odds of increased pain during their first dressing change in comparison with patients with delayed presentations (OR 0.9, $95 \%$ CI 0.8 to $0.9, \mathrm{p}<0.001)$. Based on data from this model, there is little evidence of a relationship between procedural pain levels and gender, ATSI children, place of birth, burn mechanism, anatomical burn region excluding hand and foot burns, first aid, and socioeconomic status ( $p>0.05)$.

\section{DISCUSSION}

Pain management is one of the most important aspects of burn care. Procedural pain protocols require continuous revision and reassessment to ensure optimal pain management is being provided to burn patients. ${ }^{15}$ This investigation aimed to determine if relationships existed between patient and clinical characteristics, and pain at first dressing change for children with acute burn injuries. Findings from this investigation serve as an alert for healthcare professionals to factors associated with moderate to severe pain in pediatric burn patients during their first dressing change. The identification of children with clinical characteristics associated with increased pain allows for pre-emptive implementation of pharmacological and non-pharmacological interventions for pain reduction, and the development of individualized treatment plans for these patients. Preventing the onset of severe procedural pain is the current goal-as opposed to controlling and reducing severe pain following its onset (ie, requiring a rescue dose of analgesic medication during the procedure). ${ }^{16}$ It is recommended that pediatric burn patients with the following characteristics are screened and identified before their first dressing change-so additional methods of analgesia and distraction can be implemented. These characteristics include large TBSA percentages, burns to four or more anatomical regions, baseline pain in the waiting room, receiving initial treatment at a non-burns center prior to referral, presentations outside 24 hours post-burn, hand burns, foot burns, and DDPT injuries. These burn characteristics place a child at greater odds of moderate to severe procedural pain during their first dressing change.

One potential explanation for the association between pain, TBSA, and the number of anatomical regions burned relates to spatial summation of pain. ${ }^{17}$ Spatial summation (SS) of pain is as a nociceptive phenomenon where an increase in the area of, or distance between, noxious stimuli results in greater perceived pain. ${ }^{18}$ Area-based SS can be explained through peripheral neuronal mechanisms. That is, an increase in the number of impulses from afferent nociceptors will occur when an entire receptive field is exposed to noxious stimulation, compared with when the same stimulus covers a smaller area of the receptive field. Therefore, an increase in the area of a noxious stimulus will result in an increase in the level of pain experienced. Area-based SS provides a potential mechanism for the association between increased pain and each 1\% increase in burn TBSA. This finding supports past research that also identified increased TBSA as an influential factor for procedural pain scores in pediatric burn patients $(\mathrm{p}<0.05){ }^{19}$

Distance-based SS occurs when two noxious stimuli are positioned further apart, and pain is perceived as more intense. For example, identical pairs of noxious thermal stimuli administered to the leg and abdomen with differing distances of separation $(0,5,10,20$, and $40 \mathrm{~cm})$ will be perceived with different levels of pain. ${ }^{2021}$ The level of separation between stimuli influence the perception of pain, in addition to the level of connectedness between the two noxious stimuli. That is, noxious stimuli delivered $10 \mathrm{~cm}$ apart can be perceived as connected - and the sensation of pain can radiate to regions not receiving noxious thermal stimuli. $^{20}$ Distance-based SS provides a potential mechanism for the association between increased pain and burns to four or 
Table 2 ORs from ordinal logistic regression showing bivariate (crude) relationships between procedural pain and clinical variables

\begin{tabular}{|c|c|}
\hline Independent variable & Crude $\mathrm{OR}(95 \% \mathrm{Cl})$, $\mathrm{p}$ value \\
\hline Time to QCH & $0.9(0.8$ to 0.9$),<0.001$ \\
\hline Burn size (TBSA \%) & $1.0(1.0$ to 1.2$),<0.01$ \\
\hline \multicolumn{2}{|l|}{ Baseline pain } \\
\hline Pain in waiting room & 4.9 (2.4 to 9.8$),<0.001$ \\
\hline No pain & 1.0 (reference) \\
\hline \multicolumn{2}{|l|}{ Burn depth } \\
\hline Full thickness & $5.6(2.0$ to 16.4$),<0.01$ \\
\hline DDPT & 7.8 (3.9 to 15.6$),<0.001$ \\
\hline SPT & $3.1(1.6$ to 5.9$),<0.01$ \\
\hline Superficial & 1.0 (reference) \\
\hline \multicolumn{2}{|l|}{ No of regions burned } \\
\hline $4+$ & 6.9 (1.1 to 44.5$), 0.04$ \\
\hline 3 & $2.7(1.0$ to 7.6$),<0.06$ \\
\hline 2 & $1.7(1.0$ to 3.0$),>0.05$ \\
\hline 1 & 1.0 (reference) \\
\hline \multicolumn{2}{|l|}{ Presentation type } \\
\hline Inpatient & $0.8(0.4$ to 1.7$), 0.55$ \\
\hline Outpatient & 1.7 (1.3 to 2.3$),<0.001$ \\
\hline ED & 1.0 (reference) \\
\hline \multicolumn{2}{|l|}{ Anatomical region burned } \\
\hline Hand & 1.5 (0.9 to 2.5$), 0.17$ \\
\hline Foot & $1.4(0.8$ to 2.5$), 0.25$ \\
\hline Upper limb burns & 0.9 (0.5 to 1.5$), 0.57$ \\
\hline Lower limb burns & 0.9 (0.5 to 1.5$), 0.59$ \\
\hline Head/neck & 0.7 (0.4 to 1.3$), 0.21$ \\
\hline Back & $0.6(0.3$ to 1.4$), 0.22$ \\
\hline Chest/breast & 1.0 (0.5 to 1.7$), 0.88$ \\
\hline Abdomen & 0.9 (0.5 to 1.6$), 0.61$ \\
\hline \multicolumn{2}{|l|}{ Sex } \\
\hline Male & $0.9(0.7$ to 1.1$), 0.40$ \\
\hline Female & 1.0 (reference) \\
\hline \multicolumn{2}{|l|}{ Indigenous status } \\
\hline ATSI & 0.8 (0.5 to 1.2$), 0.23$ \\
\hline Not indigenous & 1.0 (reference) \\
\hline \multicolumn{2}{|l|}{ Place of birth } \\
\hline Oceania & $0.6(0.2$ to 1.3$), 0.16$ \\
\hline Middle East & $2.1(0.5$ to 9.0$), 0.30$ \\
\hline Europe & $1.0(0.3$ to 3.0$), 0.99$ \\
\hline North America & 1.5 (0.1 to 16.5$), 0.75$ \\
\hline Africa & 0.3 (0.0 to 2.3$), 0.23$ \\
\hline Asia & 1.3 (0.6 to 3.0$), 0.51$ \\
\hline Australia & 1.0 (reference) \\
\hline \multicolumn{2}{|l|}{ Burn mechanism } \\
\hline Radiant heat & $0.6(0.2$ to 1.9$), 0.43$ \\
\hline Cooling & $1.3(0.1$ to 13.3$), 0.81$ \\
\hline Chemical & $0.7(0.3$ to 2.0$), 0.52$ \\
\hline Friction & $0.6(0.4$ to 1.2$), 0.16$ \\
\hline Flame & 1.0 (0.6 to 1.8$), 0.98$ \\
\hline Contact & 0.8 (0.6 to 1.1$), 0.22$ \\
\hline Scald & 1.0 (reference) \\
\hline \multicolumn{2}{|l|}{ First aid } \\
\hline Undocumented duration & 1.1 (0.8 to 1.4$), 0.53$ \\
\hline Inadequate & $0.8(0.4$ to 1.6$), 0.48$ \\
\hline Adequate & 1.0 (reference) \\
\hline
\end{tabular}

Continued
Table 2 Continued

Independent variable

Crude OR $(95 \% \mathrm{Cl})$, p value

a: 1.0 (reference) indicates the references group for the ordinal logistic regression. $\mathrm{b}: \mathrm{p}$ values not adjusted for multiple comparisons.

QCH, Queensland Children's Hospital; DDPT, deep dermal partial-thickness; SPT, superficial partial-thickness; TBSA, total body surface area; OPD, outpatient department.

$E D$, emergency department.

more anatomical regions. This investigation supports evidence from previous research, which also found the number of burns a patient sustained to different anatomical areas was associated with higher pain scores $(\mathrm{p}<0.01) .{ }^{22}$

Pediatric patients who first presented to a non-specialist medical center for acute burn treatment (and were later referred to the QCH Burns OPD) had increased odds of higher procedural pain during their first dressing change compared with patients who presented to the QCH ED (a burns specialist hospital) or were admitted as an inpatient at the $\mathrm{QCH}$ prior to their Burns OPD referral. This finding could be due to a number of reasons including-adult referral facilities lacking additional distraction tools and non-pharmacological interventions for pediatric patients, a lack of understanding of the analgesic requirements needed during the acute management of pediatric burns, incomplete or inadequate acute wound debridement (removal of blisters and sloughed eschar), or the use of different wound dressings (ie, silver sulfadiazine and gauze vs Mepitel and Acticoat). Moreover, time to present to the $\mathrm{QCH}$ was also a significant predictor of pain in the model-with reduced odds of increased pain for patients that presented to the $\mathrm{QCH}$ soon after sustaining their burn (within 24 hours) compared with those with delayed presentations. This finding could be due to a number of reasons similar to those mentioned previously-including challenges removing dried eschar if burns are not debrided during the acute burn period.

Studies exploring increased risk of pain for hand and foot injuries are limited, and no research providing rationalization for our findings could be identified in the literature. One potential explanation for hand and foot burns placing a child at greater odds of increased procedural pain pertains to differences between glabrous and hair-containing skin (in combination with

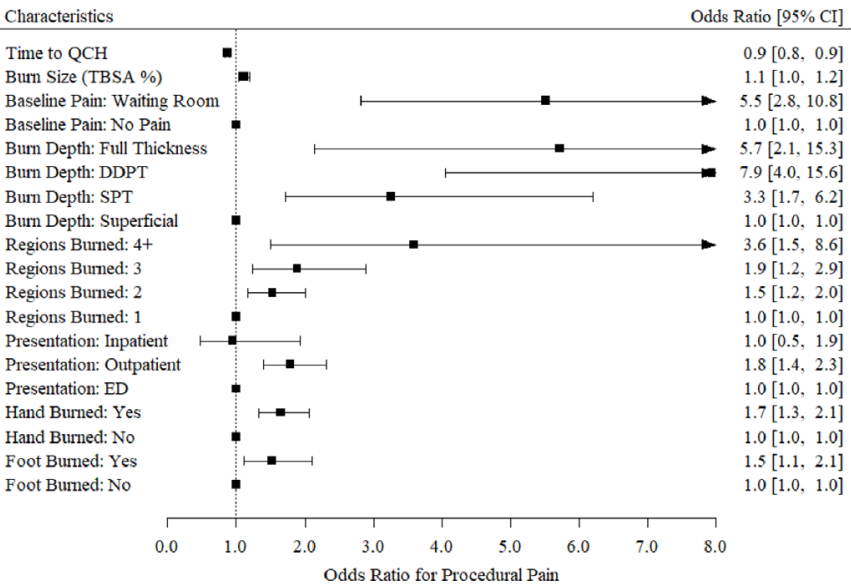

Figure 2 Multivariable relationships between procedural pain and clinical characteristics. DDPT, deep dermal partial-thickness; ED, emergency department; QCH, Queensland Children's Hospital; SPT, superficial partial-thickness; TBSA, total body surface area. 
higher order processing). Glabrous skin is hairless skin located on the palms of hands and soles of feet.

Pediatric patients with DDPT burns had almost eight times greater odds of increased pain during their first dressing change, compared with patients with superficial burns. In addition, children with full-thickness burns had more than five times greater odds of increased procedural pain. While some research suggests deeper burns are insensate and less painful than superficial injures due to the destruction of nerve afferents in the dermis and epidermis ${ }^{23}$-recent studies found no significant difference in pain scores between patients with acute isolated full-thickness burns and those with SPT burns following presentation to an adult ED. ${ }^{22}$ Most DDPT and full-thickness burns are intermediate, and will contain superficial regions with less tissue damage and intact nociceptive afferents. ${ }^{24}$ Deep burns also cause extensive tissue necrosis and edema that acts to sensitize surrounding tissue, even extending to non-burned regions. ${ }^{25}$ Peripheral sensitization resulting from inflammation, in addition to areas of intermixed depth are proposed mechanisms for the association between burn depth and pain identified in this investigation.

Results from this investigation should be interpreted with consideration of several limitations. First, because this is a retrospective cohort investigation, causal relationships could not be established-only associations. Second, due to the median age of patients treated in the Burns OPD, the FLACC scale was deemed the most appropriate tool for routine observational pain assessment during burn dressing changes. We acknowledge the limitations surrounding the use of the FLACC for procedural pain assessment and its use in older children. It is recommended that future studies investigating associations between pain and clinical features include self-report measures-as this is considered gold standard for pain assessment. Third, only outpatient data were included in this investigation. This is because patient and clinical data, in addition to dressing change pain scores, are not routinely collected for burn inpatients and uploaded to the Queensland Pediatric Burns Registry. This may limit the generalizability of our findings to a broader pediatric burn population.

\section{CONCLUSION}

Children with hand and foot burns, DDPT burn injuries, increased burn TBSA, four or more anatomical regions burned, baseline pain, and patients who received initial treatment at a non-burns center are at greater risk moderate to severe procedural pain during their first dressing change. It is important that patients with these burn characteristics are identified before their first dressing change, so additional methods of pain control and distraction can be implemented.

Acknowledgements We want to thank all the children and families for their time and participation in our research. We also wish to acknowledge and give thanks to the Multidisciplinary Burns Team at the Queensland Children's Hospital for their support in the development and implementation of this research.

Contributors BRG, MDH, and RMK conceived the research, designed the trial, and obtained research funding. MDH and SHA undertook data extraction, data cleaning, and data management. LVJ provided statistical support and conducted the formal analyses. MDH interpreted the data and wrote the draft manuscript. All authors provided critical review of the article and approved the final manuscript. MDH takes responsibility for the paper as a whole.

Funding This research received financial support in the form of a research grant awarded to The University of Queensland by Mundipharma and a Research Training Program Scholarship awarded to the primary investigator.

Disclaimer Mundipharma had no role in the development of the trial, data collection, data analysis, or interpretation of results.

Competing interests None declared.

\section{Patient consent for publication Not required.}

Ethics approval This trial received ethics approval from the Queensland Children's Health Service (Queensland Children's Hospital) Human Research Ethics Committee (approval no. HREC/16/QRCH/322) and The University of Queensland Ethics Committee (clearance no. 2017000979).

Provenance and peer review Not commissioned; externally peer reviewed. Data availability statement Data are available on reasonable request.

Open access This is an open access article distributed in accordance with the Creative Commons Attribution Non Commercial (CC BY-NC 4.0) license, which permits others to distribute, remix, adapt, build upon this work non-commercially, and license their derivative works on different terms, provided the original work is properly cited, an indication of whether changes were made, and the use is noncommercial. See: http://creativecommons.org/licenses/by-nc/4.0/.

\section{ORCID iD}

Maleea D Holbert http://orcid.org/0000-0002-9902-5122

\section{REFERENCES}

1 Stockton KA, Harvey J, Kimble RM. A prospective observational study investigating all children presenting to a specialty paediatric burns centre. Burns 2015;41:476-83.

2 Fenlon S, Nene S. Burns in children. BJA Educ 2007:7:76-80.

3 Bayat A, Ramaiah R, Bhananker SM. Analgesia and sedation for children undergoing burn wound care. Expert Rev Neurother 2010;10:1747-59.

4 Morgan M, Deuis JR, Frøsig-Jørgensen M, et al. Burn pain: a systematic and critical review of epidemiology, pathophysiology, and treatment. Pain Med 2018;19:708-34.

5 James DL, Jowza M. Principles of burn pain management. Clin Plast Surg 2017:44:737-47

6 Brown NJ, Kimble RM, Rodger S, et al. Play and heal: randomized controlled trial of Ditto ${ }^{\mathrm{TM}}$ intervention efficacy on improving re-epithelialization in pediatric burns. Burns 2014:40:204-13.

7 Brown NJ, Kimble RM, Gramotnev G, et al. Predictors of re-epithelialization in pediatric burn. Burns 2014:40:751-8

8 Dauber A, Osgood PF, Breslau AJ, et al. Chronic persistent pain after severe burns: a survey of 358 burn survivors. Pain Med 2002;3:6-17.

9 De Young AC, Kenardy JA, Cobham VE, et al. Prevalence, comorbidity and course of trauma reactions in young burn-injured children. J Child Psychol Psychiatry 2012;53:56-63.

10 Willis MHW, Merkel SI, Voepel-Lewis T, et al. FLACC behavioral pain assessment scale: a comparison with the child's self-report. Pediatr Nurs 2003;29:195-8.

11 Merkel S, Voepel-Lewis T, Malviya S. Pain assessment in infants and young children: the FLACC scale: a behavioral tool to measure pain in young children. Am J Nurs 2002; 102:55-8.

12 Nilsson S, FINNSTRM B, Kokinsky EVA. The FLACC behavioral scale for procedural pain assessment in children aged 5-16 years. Pediatr Anesth 2008;18:767-74.

13 World Health Organization. Cancer pain relief: with a guide to opioid availability. 2nd edn. Geneva, Switzerland: WHO, 1996.

14 Alschuler KN, Jensen MP, Ehde DM. Defining mild, moderate, and severe pain in persons with multiple sclerosis. Pain Med 2012;13:1358-65.

15 Ratcliff SL, Brown A, Rosenberg L, et al. The effectiveness of a pain and anxiety protocol to treat the acute pediatric burn patient. Burns 2006;32:554-62.

16 Wells N, Pasero C, McCaffery M. Advances in patient safety: Improving the quality of care through pain assessment and management. In: Patient safety and quality: an evidence-based Handbook for nurses. Rockville (MD): Agency for Healthcare Research and Quality, 2008: 469-89.

17 Curatolo M. Spatial summation of pain and its meaning to patients. Scand J Pain 2017:17:116-7.

18 Price DD, McHaffie JG, Larson MA. Spatial summation of heat-induced pain: influence of stimulus area and spatial separation of stimuli on perceived pain sensation intensity and unpleasantness. J Neurophysio/ 1989;62:1270-9.

19 de Jong AEE, Bremer M, van Komen $\mathrm{R}$, et al. Pain in young children with burns: extent course and influencing factors. Burns 2014:40:38-47.

20 Quevedo AS, Coghill RC. Filling-in, spatial summation, and radiation of pain: evidence for a neural population code in the nociceptive system. J Neurophysiol 2009;102:3544-53.

21 Holbert MD, Pedler A, Camfermann D, et al. Comparison of spatial summation properties at different body sites. Scand J Pain 2017;17:126-31.

22 Singer AJ, Beto L, Singer DD, et al. Association between burn characteristics and pain severity. Am J Emerg Med 2015;33:1229-31.

23 Gamst-Jensen $\mathrm{H}$, Vedel PN, Lindberg-Larsen VO, et al. Acute pain management in burn patients: appraisal and thematic analysis of four clinical guidelines. Burns 2014;40:1463-9.

24 Atchison NE, Osgood PF, Carr DB, et al. Pain during burn dressing change in children: relationship to burn area, depth and analgesic regimens. Pain 1991;47:41-5

25 Junger $\mathrm{H}$, Moore AC, Sorkin LS. Effects of full-thickness burns on nociceptor sensitization in anesthetized rats. Burns 2002;28:772-7. 